\title{
Forelimb Amputation and Long-Term Follow-Up in a Female Donkey
}

\author{
Antônio Carlos Lopes Câmara', Igor Louzada Moreira' \& Eraldo Barbosa Calado²
}

\begin{abstract}
Background: Limb amputation may be a life-saving procedure for animals and minimally impact their comfort and quality of life, as previously reported in pets. This procedure is an appropriate alternative to euthanasia when catastrophic injury to a limb prevents its successful restoration. In horses, limb amputation has been performed for the past 40 years. Although in the reviewed literature there are no scientific reports of limb amputation in donkeys. This paper aimed to report a successful forelimb amputation and long-term follow-up in a female donkey.

Case: A 10-month-old and $95 \mathrm{~kg}$ female Nordestino donkey was evaluated for a wound with bone exposition on the left forelimb. Physical examination revealed that the donkey was alert with moderate body condition score, tachycardia, tachypnea, and a medial wound revealing the necrotic aspect of the left radius. Radiographic examination presented Salter-Harris type 1 exposed radius fracture. Hematology revealed normocytic and normochromic anemia, and hyperfibrinogenemia. Due to the catastrophic injury and no forecast for building a prosthesis, the donkey underwent general anesthesia for left forelimb amputation through the midhumerus diaphysis. A fish-mouth skin incision was performed on the midhumerus, the underlying musculature was dissected and the vessels ligated until the bone could be accessed. Then, a sterile saw wire was used to transect it, and bone edges were rounded. Muscle and fascia were harvested with the skin to provide additional padding at the end of the stump. Postoperatively, the donkey was submitted to broad spectrum antibiotic, antiinflammatory and analgesic therapy, and tetanus prophylaxis. Preventative treatment for overload laminitis included frog support pads and resting leagues on all three legs. No short-term complications were observed and the donkey made a full recovery. Long-term complications included fistula formation on the stump, and distal interphalangeal joint hyperflexion, probably by weight overload causing acquired deep digital tendon contracture on the right forelimb. Currently, eight years (96-months) past surgery, the donkey is still well adapted and has a good quality of life even without a limb prosthesis.

Discussion: Traditionally, catastrophic leg injury in equids often requires euthanasia. Currently, limb amputation is becoming more accepted by owners and a viable life salvage option. In horses, limb amputation by disarticulation using a caudal flap technique is the most usual surgical technique, but presents some chronic complications, such as osteomyelitis of the stump, pressure sores from the prosthesis and contralateral limb failure. Although not commonly performed in equids, limb amputation through the midhumerus diaphysis was well accepted by the donkey. In horses, survival rate reached $50 \%$ and $32.5 \%$ at 18 and 24 months after amputation with prosthetic fitting, respectively. The donkey herein reported is still alive and presenting a good life quality at 96-months post-surgery, even without a forelimb prosthesis. This kind of feature has been only reported in a Samba deer followed-up for 10-months, showing good corporal condition, walking and even running at slow speed, using three legs. To the authors knowledge this is the first scientific report of limb amputation in a donkey. We emphasize that documenting more limb amputation in equids is essential to obtain conclusions about the prognosis, life expectancy and expected quality of life.
\end{abstract}

Keywords: adaptation, amputation, Salter-Harris fracture, donkey. 


\section{INTRODUCTION}

Limb amputation in horses has been performed for the past four decades $[2,4,6,8,9]$. Previous reports indicate that, in cases where fracture configuration, impaired circulatory status, or sepsis render traditional means of limb repair hopeless, amputation should be considered as a viable option to euthanasia [9]. In the reviewed literature, there are no scientific reports of limb amputation in donkeys. Therefore, this paper aimed to report a successful forelimb amputation and long-term follow-up in a female donkey.

\section{CASE}

A 10-month-old and $95 \mathrm{~kg}$ female Nordestino donkey was evaluated due to a wound with bone exposition on the left forelimb. It was reported that the donkey was a wandering animal and was hit by a car two days earlier, and did not receive any prophylactic or therapeutic treatment. Physical examination revealed that the donkey was alert with moderate body condition score (scale 4/9), tachycardia (72 beats per minute), and tachypnea ( 32 breathes per minute). The left forelimb presented a medial wound revealing the necrotic aspect of the radius (Figure 1A), causing grade 5/5 lameness (Figure 1B). Soft tissue swelling and necrosis were also evident. Radiographic examination presented Salter-Harris type 1 exposed radius fracture. Hematology presented normocytic and normochromic anemia (PCV: 27\%; RBC: 5.8 million $/ \mathrm{mm}^{3}$ and hemoglobin: $8.7 \mathrm{~g} / \mathrm{dL}$ ), and hyperfibrinogenemia $(6.0 \mathrm{~g} / \mathrm{L})$. Serum biochemistry profiles (urea, creatinine, albumin, total plasma protein and the activities of the enzymes aspartate aminotransferase and glutamyl transferase) were within normal limits.

The donkey underwent general anesthesia in right lateral recumbence for left forelimb amputation. After the hoof was wrapped with a plastic bag, the proximal portion of the limb was clipped and prepared aseptically. Left brachial plexus was blocked with ropivacaine ${ }^{1}\left(0.6 \mathrm{mg} \cdot \mathrm{kg}^{-1}\right)$, and a tourniquet was placed as cranially as possible on the humerus. Limb amputation was performed through the midhumerus diaphysis, following the recommendations of Desrochers et al. [3]. A fish-mouth skin incision was performed on the midhumerus (the length of each flap was equal to three-fourths of the diameter of the limb at the level of the amputation). The underlying musculature was dissected and the vessels ligated until the midhumerus could be accessed. Then, a sterile saw wire ${ }^{2}$ was used to transect it, and bone edges were rounded. Muscle and fascia were harvested with the skin to provide additional padding between the bone and the skin at the end of the stump. Skin was closed with simple, interrupted, 0 nylon sutures. During surgery a blood transfusion $(2 \mathrm{~L})$ was made because of the already existing anemia and bone marrow blood loss.

Postoperatively, the donkey was submitted to broad spectrum antibiotic association (benzathine penicillin ${ }^{3}$ : 40,000 UI.kg ${ }^{-1}$, intramuscularly [IM], q48h, 4 doses; and gentamicin ${ }^{4}: 4$ mg.kg ${ }^{-1}$, intravenously [IV], once a day [s.i.d.], 7 days), and tetanus prophylaxis. Anti-inflammatory (flunixin meglumine ${ }^{5}: 1.1$ mg.kg-1 ${ }^{-1}$ IV, s.i.d., 3 days) and analgesic (ketamine $^{6}: 0.2 \mathrm{mg} \cdot \mathrm{kg}^{-1}$, IM, 3 times a day, 5 days) therapy was also performed. Preventative treatment for overload laminitis was initiated and included frog support pads and

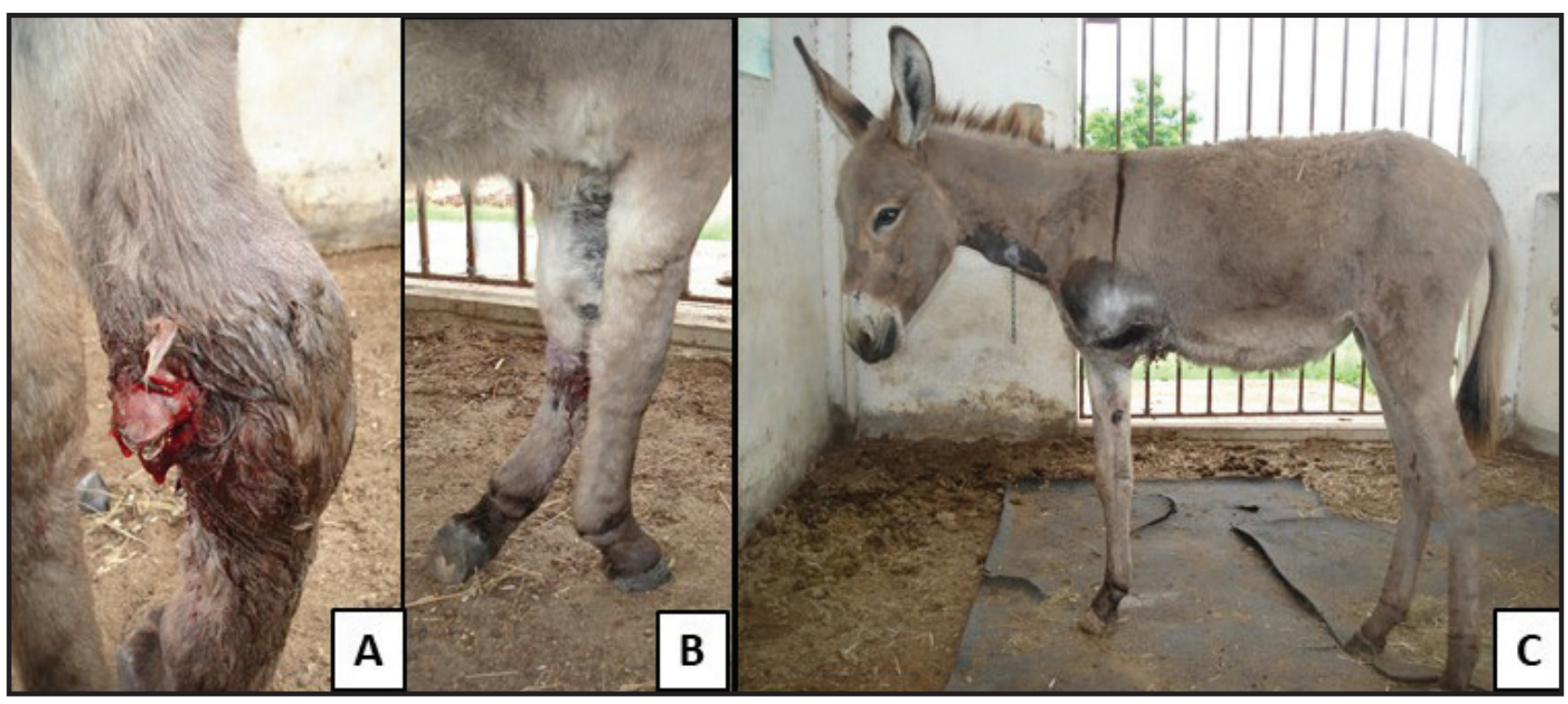

Figure 1. A- Female donkey presenting a medial wound revealing the necrotic aspect of the left radius (Salter-Harris type 1 fracture); B- Left forelimb with grade 5/5 lameness; C- Immediately after surgery, the donkey weight bearing well on three legs. 


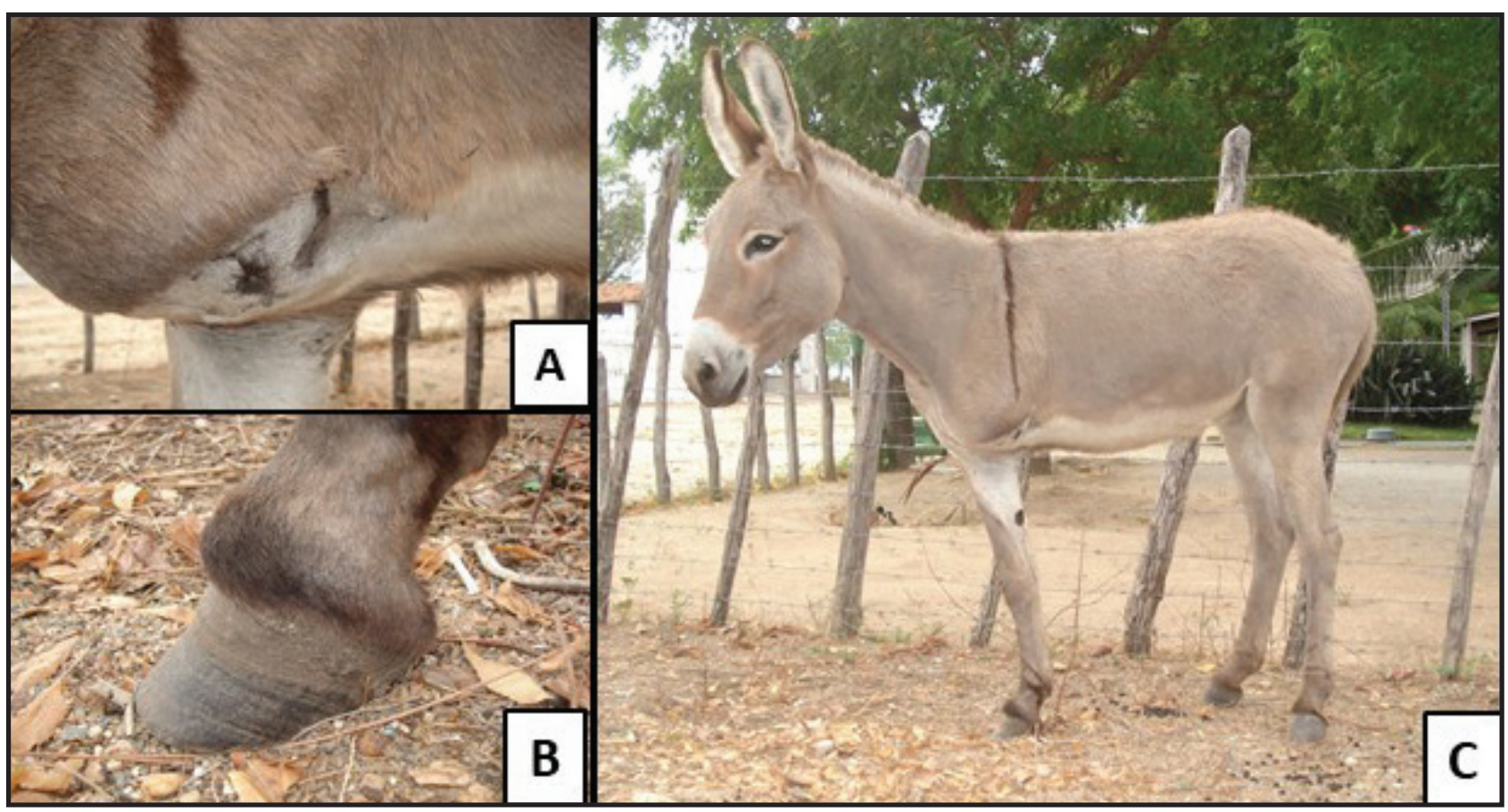

Figure 2. A- Healed fistula on the stump (5-months post-amputation); B- Note hoof capsule distortion, prominent growth rings, toe flaring toward and dorsal projection of the extensor process forming a shelf in the front of the coronet as consequence of deep digital tendon contracture on the right forelimb; C- Eight years (96-months) past surgery, the donkey is still well adapted and has a good life quality even without a limb prosthesis.

resting leagues on all three legs. Surgical wound was cleaned with $0.2 \%$ chlorhexidine solution twice daily for 15 days until stiches removal. No short-term complications were observed and the donkey made a full recovery (Figure 1C).

The donkey was adopted and remained receiving the adequate care to prevent overload laminitis. About 3 months after surgery, the stump presented a fistula draining serous-bloody content, that was treated by daily local antiseptic and antibiotic lavage $(0.2 \%$ gentamicin and chlorhexidine solution), healing in about 60 days (Figure 2A). Corrective hoof trimming had to be performed every three months because of toe overgrowth. In the first year post surgery, distal interphalangeal joint (DIJ) hyperflexion causing acquired deep digital tendon contracture on the right forelimb was noted, probably due to weight overload. This acquired flexural deformity caused distortion of the hoof capsule, prominent growth rings, toe flaring toward and dorsal projection of the extensor process forming a shelf in the front of the coronet (Figure 2B). It was also recommended that the donkey did not get pregnant to preclude contralateral limb failure. Currently, 8 years (96-months) past surgery, the donkey is still well adapted and has a good life quality even without a limb prosthesis (Figure 2C).

\section{DISCUSSION}

Traditionally, catastrophic leg injury in equidae often required euthanasia [4]. Currently, limb amputation is becoming more accepted by owners and a viable alternative to preclude euthanasia [2]. It can be more than a life-sparing procedure and enable a productive life, since mares and stallions have continued breeding following partial limb amputation [2,4].

Limb amputation may be done either through the diaphysis or by disarticulation [3]. In horses, limb amputation by disarticulation using a caudal flap technique is the most usual $[6,8,9]$. This technique combines suturing the suspensory ligament and flexor tendons to the extensor tendons, and the incorporation of frog tissue in the stump to minimize chronic complications, such as osteomyelitis and pressure sores. Additionally, disarticulation provides a wider surface for weight bearing on the prosthesis, reduced hemorrhage, and faster soft tissue healing with less risk of dehiscence because of the absence of sharp bone margins $[3,6]$. All the aforementioned benefits improve the successful rate for wearing a prosthetic $[2,4,6,8,9]$. In our case there was no forecast for purchasing or manufacturing a prosthesis, and limb amputation through the midhumerus diaphysis was chosen. Although not commonly performed in equids, amputation through the diaphysis has been reported through the distal third of the tibia, proximal radius-ulna, midfemur, midhumerus, distal femur, proximal metatarsi, and distal metacarpus in cattle [3]. In this donkey, this technique's main disadvantage was trans-surgical hemorrhage since bone marrow was exposed; even requiring blood transfusion. 
Since no prosthesis was used, the most important long-term complication was contralateral limb flexural deformity. Acquired deformities develop after birth, and their cause is likely multifactorial. Although the most important causes are nutritional and mineral imbalances; painful limb conditions, such as chronic disproportionate limb loading related to lameness, forelimb grazing posture preferences that influence limb loading discrepancies, and hoof wear patterns (forward foot vs rearward foot), neuromuscular dysfunction or hoof abscessation may also cause the problem [1]. Equines with acquired flexural deformity may lack lameness and should travel short distances in their daily activities. Once flexural deformity of the DIJ becomes chronic, the sequel can be significant hoof capsule distortion, separation at the white line, and flaring of the hoof wall, resulting in deep digital flexor tendonitis, degenerative DIJ disease, osseous remodeling, demineralization or septic osteitis of distal phalanx, which greatly reduces the long-term survival. In cases where there is no pain, the animal should undergo frequent trimming to alleviate distortions of the foot and problems with the distal phalanx. [1,5]. In this report, the donkey seems to have a good quality of life, since it stays lying down only for short periods, sometimes even walks fast through the paddock, and always ate very well.

One of the major limitations of the use of prosthetic devices in horses is the high complication rate $[2,4]$. Perioperative complications include fracture upon recovery from anesthesia, wound dehiscence and poor patient acceptance [6]. Most failures have occurred as a result of chronic complications such as osteomyelitis of the stump, pressure sores from the prosthesis and contralateral limb failure $[2,4,6,8,9]$. In previous reports, survival rate achieved 50\% (9/18) and 32.5\% (8/26) until 18 months [2] and more than 24 months after amputation [8]. Kelmer et al. [4] reported a limb amputation $10 \mathrm{~cm}$ distal to the tarsometatarsal joint in a 20y-old Arabian-cross stallion, that presented several pressure sores and lameness episodes until euthanasia at 24-months post-surgery. Additionally, a 10-year-old Shetland pony amputated at the level of the metacarpophalangeal joint was still alive on the follow-up at 26 months after surgery [6].

To the authors knowledge this is the first scientific report of limb amputation in a donkey. Moreover, in this report no prosthesis was used and the donkey is still alive and presenting a good life quality at 96 -months ( 8 years) post-surgery. This kind of feature has been only reported in a Samba deer followed-up for 10-months, showing good corporal condition, walking and even running at slow speed using three legs [7]. The light weight and rusticity of the donkey in this report were probably positive factors in the avoidance of complications and good outcome. We emphasize that documenting more limb amputation in equids is essential to obtain conclusions about the prognosis, life expectancy and expected quality of life.

\section{MANUFACTURERS}

${ }^{1}$ Cristal Pharma. Contagem, MG, Brazil.

${ }^{2}$ Jørgen Kruuse A/S. Langeskov, Denmark.

${ }^{3}$ União Química Farmacêutica Nacional S/A. Embu-Guaçu, SP, Brazil.

${ }^{4}$ Ceva Saúde Animal Ltda. Paulínia, SP, Brazil.

${ }^{5}$ Uzinas Chemicas Brasileiras. Jaboticabal, SP, Brazil.

${ }^{6}$ Syntec do Brasil. Santana de Parnaíba, SP, Brazil.

Declaration of interest. The authors report no conflicts of interest. The authors alone are responsible for the content and writing of the paper.

\section{REFERENCES}

1 Caldwell F.J. 2017. Flexural deformity of the distal interphalangeal joint. Veterinary Clinics of North America: Equine Practice. 33(2): 315-330.

2 Crawley G.R., Grant B.D., Krpan M.K. \& Major M.D. 1989. Long-term follow-up of partial limb amputation in 13 horses. Veterinary Surgery. 18(1): 52-55.

3 Desrochers A., St-Jean G. \& Anderson D.A. 2014. Limb amputation and prosthesis. Veterinary Clinics of North America: Food Animal Practice. 30(1): 143-155.

4 Kelmer G., Steinman A., Levi O. \& Johnston D.E. 2004. Amputation and prosthesis in a horse: short- and long-term complications. Equine Veterinary Education. 16(5): 235-241.

5 McGladdery A.J. 1992. Three cases of acquired flexural deformity of the distal interphalangeal joint in growing foals on a stud farm. Equine Veterinary Education. 4(4): 173-176.

6 Moulin N., Schramme M., François I., Castelijns G. \& Belluco S. 2018. Long-term outcome of treatment of a squamous cell carcinoma of the foot by amputation of the distal limb in a pony. Equine Veterinary Education. https:// doi.org/10.1111/eve.12925 
A.C.L. Câmara, I.L. Moreira \& E.B. Calado. 2019. Forelimb Amputation and Long-Term Follow-Up in a Female Donkey.

7 Singh K., Kumar A., Mahajan S.K. \& Saini N.S. 2010. Successful forelimb amputation procedure on a sambar deer (Cervus unicolor niger). Journal of Wildlife Rehabilitation. 30(2): 21-24.

8 Vlahos T.P. \& Redden R.F. 2005. Amputation of the equine distal limb: indications, techniques and long-term care. Equine Veterinary Education. 17(4): 212-217.

9 Vlahos T.P., Grant B.D. \& Hawkings H.A. 2010. How to perform amputation of the equine limb using a caudal flap technique. AAEP Proceedings. 56: 187-191. 